\title{
Research on Dihydrate Calcium Sulphate Crystallization during the Production of Phosphoric Acid by Wet Process
}

\author{
Huirong Du ${ }^{1,2}$, Anyin Chen ${ }^{1,2 *}$, Li Yang1 \\ ${ }^{1}$ Department of Chemistry and Chemical Engineering, Sichuan University of Arts and Science, Dazhou, China \\ ${ }^{2}$ Key Laboratory of Exploitation and Study of Distinctive Plants in Education Department of Sichuan Province, \\ Sichuan University of Arts and Science, Dazhou, China \\ Email: anyinchen@163.com
}

Received 24 October 2014; revised 28 November 2014; accepted 10 December 2014

Copyright (C) 2014 by authors and Scientific Research Publishing Inc.

This work is licensed under the Creative Commons Attribution International License (CC BY). http://creativecommons.org/licenses/by/4.0/

(c) (i) Open Access

\begin{abstract}
Simulate the production of phosphoric acid by wet process is conducted in batch experiments facilities by adopting phosphorus concentrate as raw material from Wengfu, Dazhou city, with a view to study sulfuric acid concentration, reaction time, crystallization temperature of calcium sulfate, active additive, the influence of seed crystal on the size and shape of calcium sulfate crystal under conditions of Wet process phosphoric acid productive process. The research results show that under the conditions of the liquid-solid ratio of 4:1, the sulfate acid stoichiometric ratio of 1.1 , phosphoric acid mass fraction of $21 \%$, the crystallization temperature of $75^{\circ} \mathrm{C}$, crystallization time of $120 \mathrm{~min}$, when ( $\left.\mathrm{NH}_{4}\right)_{2} \mathrm{SO}_{4}$ taken as active additives, Mg-doped calcium sulphate crystal as crystal seed can obtain rodlike crystallization of stability, thickness, uniformity.
\end{abstract}

\section{Keywords}

Calcium Sulfate, Active Additives, Crystal Seed, Crystallization

\section{Introduction}

At present, phosphoric acid industry have given priority to wet process production, and based on different forms of calcium sulfate crystal compound, wet process phosphoric acid production can be divided into dihydrate, semihydrate, semihydrate-dihydrate, dihydrate-semihydrate, anhydrate and other process. Because of strong adaptability of dihydrate phosphoric acid to phosphate rock, with relatively loose production condition, more than

\footnotetext{
${ }^{*}$ Corresponding author.
}

How to cite this paper: Du, H.R., Chen, A.Y. and Yang, L. (2014) Research on Dihydrate Calcium Sulphate Crystallization during the Production of Phosphoric Acid by Wet Process. Journal of Materials Science and Chemical Engineering, 2, 7-11. 
90\% production of wet process phosphoric acid adopts dihydrate process, and is, production of wet process phosphoric acid can be said to be a crystal technology in a sense, for small calcium sulphate crystal would restrict production and yield enhancement due to the difficulty of filtering and washing; however, when crystallization is carried on the particle surface of phosphate rock, a large number of small calcium sulfate crystallizations would be generated, depositing on its surface then turning into solid film of poor permeability, which increases the diffusion resistance of acid to the surface of phosphate rock, thus reducing the reaction rate, or even terminating reaction process. Therefore, the normal production of wet process phosphoric acid production can be ensured only by keeping calcium sulfate crystallization in good condition. There are two specific requirements for the calcium sulfate crystallization process: stable shape of calcium sulfate crystal; thick and neat crystal particles with good filtering performance and washing effect [1] [2]. At the same time, the phosphorus gypsum with large crystal particles and fewer impurities are also required when served as additive in papermaking industry and plastic industry, and in this way calcined $\beta$-gypsum can reach the strength standard. Therefore, the massive calcium sulfate crystallization produced is the prerequisite to guarantee steady production, normal application for downstream users.

In order to obtain stable, bulky, uniform crystallization, a lot of studies have been conducted at home and abroad [3] [4], improve the reaction speed and elevate the filtration strength of phosphoric acid production. Western oil companies in the United States adopt the organic sulfonic acid and sulfonate of tall oil to control nuclear generation of semihydrate; Japanese like Wei Jingzhi adopt the alkyl benzene sulfonic acid to refrain the formation of dihydrate crystal nucleus, so as to raise metastability of semihydrate.

Shape and size of calcium sulfate crystal is associated with the control of various conditions in the process of crystallization, such as crystallization temperature, concentration of free acid, residence time, stirring speed, etc. In addition, active additive can be added in the process of crystallization, which also effectively improves the crystal growth rate, thus making the crystal grow up rapidly [5]. In order to improve the reaction conditions, to ascend the reaction speed and increase the yield of phosphorus, and at the same time, to obtain stable, bulky, uniform and neat crystallization, the experiment have studied of technological parameters of wet process phosphoric acid and effects of active additives and crystal seed on size and shape of calcium sulfate crystal.

\section{Experimental Part}

\subsection{Materials and Instruments}

Phosphorus concentrate(Wengfu, Dazhou city), phosphoric acid (AR), concentrated sulfuric acid, reaction kettle of electric heating, drying oven, vacuum pump, electric mixer, the suction filter bottle, magnetic stirrer.

\subsection{Experimental Process}

This experiment adopts the simulating production process of Wengfu phosphoric acid, with acidolysis reaction of phosphate rock powder completed in glass heating reaction kettle, and reaction kettle ought to be configured a stirrer to mix with constant stirring, with electric heating. Concentrated sulfuric acid and sour regurgitation should be added with burette of constant speed, and ground phosphate rock can be added by artificial weighing with multiple batches, once every 3 min. Acidolysis slurry should be sampled every $30 \mathrm{~min}$, by virtue of Buchner funnel vacuum suction filter, with vacuum control at 0.04 to $0.06 \mathrm{MPa}$. Filter cake using quadratic countercurrent washing, with washing water addition about 2 times the ore quantity, at the same time, according to the quality score of phosphoric acid in reaction slurry, washing water volume can be adjusted.

\subsection{Experiment Method}

According to the analysis of data and related material balancing calculation before the experiment, experimental conditions can be preliminary predicted, with provisional technical index. After experiments to explore the main technological conditions, continuous acidolysis experiment of single factor condition is carried out by batch charging. The main conditions affecting calcium sulfate crystallization process are optimized. In the experiment, with the other process conditions unchanged, one factor in appropriate range is changed. Filtration can be conducted after reaching the predetermined reaction time, and then leaching residue is washed by anhydrous ethyl alcohol, after drying for 3 hours under $65^{\circ} \mathrm{C}$, and finally particle size determination and morphology characterization can be figured out. 


\section{Results and Discussion}

\subsection{Effect of Sulfuric Acid Concentration}

Take five samples of sulfuric acid with its stoichiometric ratio to $100 \mathrm{~g}$ phosphorus concentrate of $0.9,1.0,1.1$, 1.2, and 1.3 are diluted into $400 \mathrm{ml}$ separately, experiment is conducted according to 1.2 experimental processes, with the above solution heated to $75^{\circ} \mathrm{C}$ and for 2 hours insulation, and then samples are taken by vacuum filtration. After comparison of experimental results (Figure 1), it has manifested that larger crystals of easy-washing and filtering can be formed when sulfuric acid stoichiometric ratio stabilizes at 1.1. It may due to the fact that little excessive sulfuric acid can reduce the degree of super saturation of the solution, slowing down the rate of crystal nucleus formation, thus creating good conditions for the growth of the crystal. When sulfuric acid stoichiometric ratio is increased to 1.3 , there is no obvious change in the size of the crystal, while the crystal assumes to be the acicular, which may cause adverse effects on the practical production, such as the difficulty to wash or inclusion phenomena. Thus, sulfate stoichiometric ratio of 1.1 should be chosen in the production process.

\subsection{Effect of Crystallization Temperature}

Under the conditions of the liquid-solid ratio of 4:1, the sulfate acid stoichiometric ratio of 1.1, phosphoric acid mass fraction of $21 \%$, the effect of temperature on the crystallization morphology of calcium sulfate is investigated. After comparison of experimental results, it has been found that higher reaction temperature results in lower viscosity of the solution, which contributes to the spread of the solute molecules and speed up the reaction, thus improving crystallization and reducing the loss of $\mathrm{P}_{2} \mathrm{O}_{5}$ among lattices. With the increase of reaction temperature, the average crystal size increases gradually, but there is no obvious changes in its shape, all in rodlike shape. But when the temperature is too high, it can increase the risk of material corrosion and the solubility of impurity in acid solution, so it is appropriate to control the temperature at $75^{\circ} \mathrm{C}$ in actual production.

\subsection{Effect of the Crystallization Time}

Under the conditions of the liquid-solid ratio of 4:1, the sulfate acid stoichiometric ratio of 1.1, phosphoric acid mass fraction of $21 \%$, the crystallization temperature of $75^{\circ} \mathrm{C}$, investigation of the influence of crystallization time (30 min, $60 \mathrm{~min}, 90 \mathrm{~min}, 120 \mathrm{~min}, 150 \mathrm{~min}, 180 \mathrm{~min}$ ) on the crystallization morphology of calcium sulphate has been made respectively. Experimental results show that the crystallization time 120 min can make crystal bulky, uniform. If the residence time is too short, then the crystal nucleus density is very big, thus generating tiny crystals of poor quality and uniformity; with the increase of residence time, crystals become large and uniform; but when the time is too long, crystal become tiny again, which may lead to a large number of formation of quadratic crystal nucleus, resulting in significantly decreased crystal growth rate. By comprehensive considerations of production time and the yield, the crystallization time should be appropriately controlled at 120 $\min$.

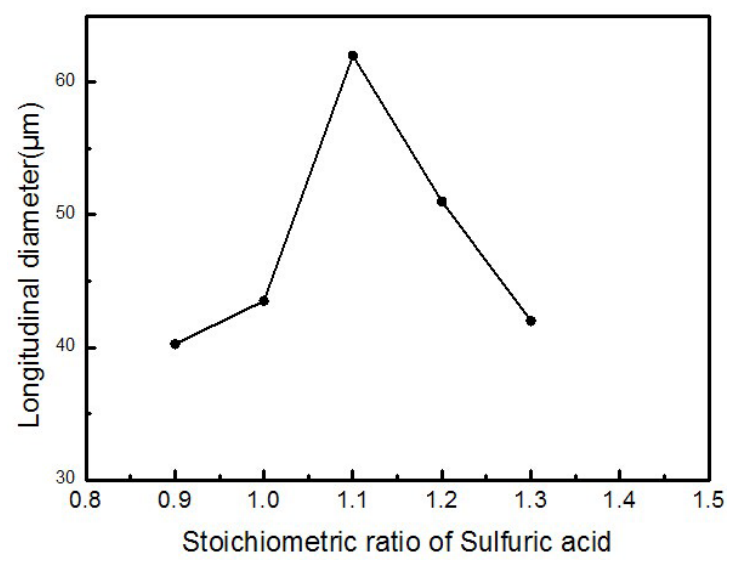

Figure 1. Effect of sulfuric acid concentration. 


\subsection{Effect of Shape the Surface Active Agent on Calcium Sulfate Crystal}

Under the conditions of the liquid-solid ratio of $4: 1$, the sulfate acid stoichiometric ratio of 1.1 , phosphoric acid mass fraction of $21 \%$, the crystallization temperature of $75^{\circ} \mathrm{C}$, crystallization period of $120 \mathrm{~min}$, six kinds of typical surface active agent are added respectively in the process of crystallization: nonionic surfactants of polyacrylamide, anionic surfactant of sodium dodecyl benzene sulfonic acid, nonionic surfactants of polyethylene glycol, active diatomite, cationic surfactant active agent of CTAB, $\left(\mathrm{NH}_{4}\right)_{2} \mathrm{SO}_{4}$. Experimental results show that surfactant generally makes the crystal growth rate accelerated, the crystal size also increased significantly, in which $\left(\mathrm{NH}_{4}\right)_{2} \mathrm{SO}_{4}$ and the active diatomite is most obvious.

\subsection{Preparation of Crystal Seed and Its Effect}

Calcium sulfate crystal of magnesium-doped, iron-doped, aluminum-doped and zinc-doped is prepared by ourselves as crystal seed, the specific method is: to add $2 \%$ magnesium, iron, aluminum, zinc nitrate into the aqueous solution of the pure calcium sulphate crystal by analysis, to filtrate after crystallizing for 2 hours under the temperature of $40^{\circ} \mathrm{C}$, and to get the corresponding crystal seed after drying under the temperature of $45^{\circ} \mathrm{C}$. crystal seed should be added by $0.5 \%$ mineral powder in the process of acid decomposition of phosphate rock, in which magnesium-doped crystal seed can get bulky (Figure 2), uniform, neat rod-shaped crystallization, with 25\% crystallization time shortened. When crystal seed of $\mathrm{CaSO}_{4}$ not added, average width of calcium sulfate crystal particle is $9 \mu \mathrm{m}$, the average length of $55 \mu \mathrm{m}$, which is a fine needle crystal; when magnesium-doped calcium sulfate crystal seed added, doped magnesium chloride expands the specific surface area of crystal seed, reducing

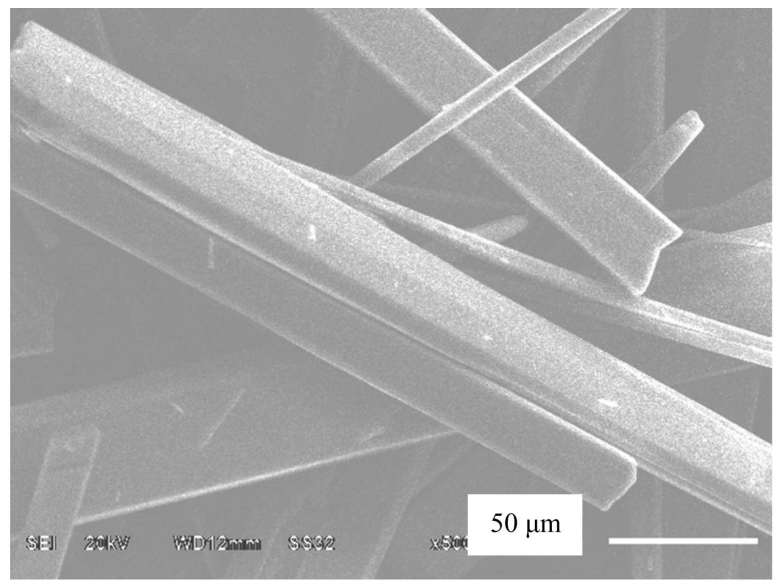

(a)

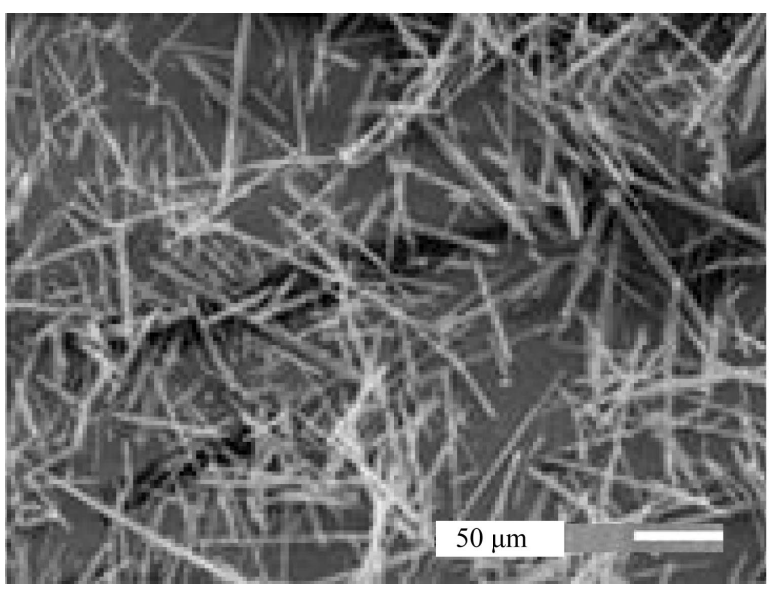

(c)

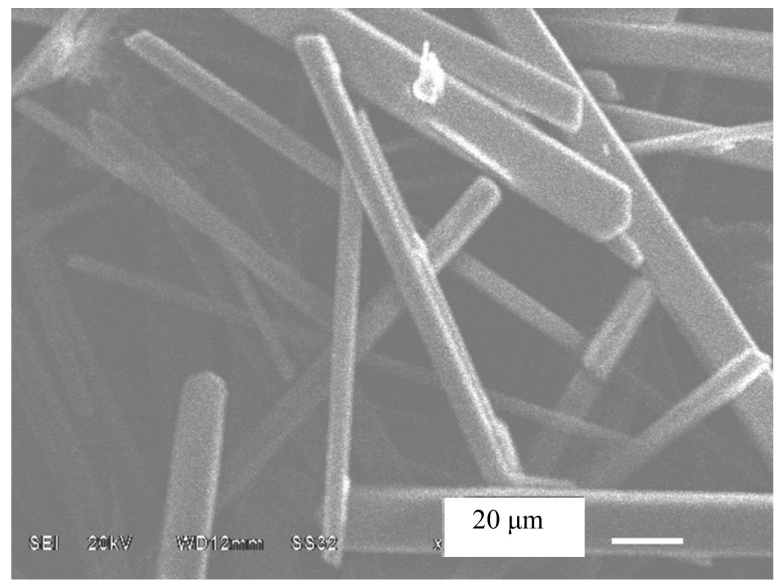

(b)

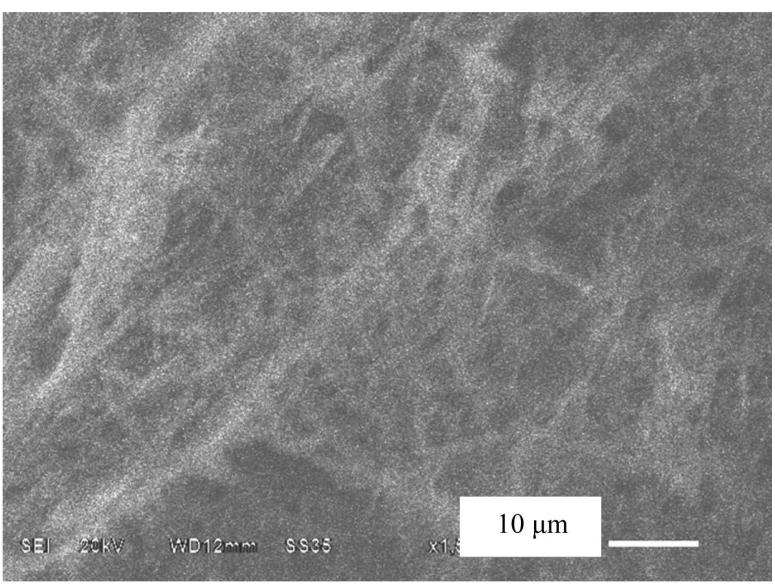

(d)

Figure 2. Effect of crystal seed (a) Magnesium-doped; (b) Iron-doped; (c) Aluminum-doped; (d) Zinc-doped. 
the free energy of particle nucleation, effectively preventing too high degree of solution super saturation, which is more conducive to the growth of crystal, thus gradually growing up to be a thick crystallization of rod like shape.

\section{Conclusion}

Regarding phosphorus concentrate from Wengfu, Dazhou city as raw material, we have optimized the process parameters through adoption of method of single factor experiment conditions by simulating wet process phosphoric acid production process, filtrate the surface active agent, with crystal seed prepared and filtrated, thus obtaining satisfactory crystallization results. Under the conditions of the liquid-solid ratio of 4:1, the sulfate acid stoichiometric ratio of 1.1 , phosphoric acid mass fraction of $21 \%$, the crystallization temperature of $75^{\circ} \mathrm{C}$, crystallization time of $120 \mathrm{~min}$, when $\left(\mathrm{NH}_{4}\right)_{2} \mathrm{SO}_{4}$ is taken as active additives, Mg-doped calcium sulphate crystal as a crystal seed can obtain rodlike crystallization of stability, thickness, uniformity.

\section{Acknowledgements}

This study was financially supported by the scientific research fund of Sichuan Provincial Education Department (12ZA146, 12ZB118); the scientific research fund of Dazhou Science \& Technology Bureau.

\section{References}

[1] Anirudhan, T.S. and Ramachandran, M. (2006) Adsorptive Removal of Tannin from Aqueous Solutions by Cationic Surfactant-Modifled Bentonite Clay. Journal of Colloid and Interface Science, 299, 116-124. http://dx.doi.org/10.1016/j.jcis.2006.01.056

[2] Jaw, K.-S., et al. (2001) The Thermal Decomposition Behaviors of Satiric Acid, Paraffin Wax and Poly-Vinyl Butyric. Thermochimica Acta, 367, 165-168.

[3] Shindo, H., et al. (2008) Stability of Surface Atomic Structures of Ionic Crystals Studied by Atomic Force Microscopy Observation of Various Faces $\mathrm{CaSO}_{4}$ Crystal in Solution. JVST B, 14, 1365-1368.

[4] Follner, S., et al. (2012) The Setting Behaviour of $\alpha$ - and $\beta$ - $\mathrm{CaSO}_{4} \cdot 0.5 \mathrm{H}_{2} \mathrm{O}$ as a Function of Crystal, Structure and Morphology. Crystal Research and Technology, 37, 1075-1087. http://dx.doi.org/10.1002/1521-4079(200210)37:10<1075::AID-CRAT1075>3.0.CO;2-X

[5] Boeyens, J.C.A. and Ichharam, V.V.H. (2002) Redetermination of the Crystal Structure of Calcium Sulfate Dehydrate. Zeitschrift fur Kristallographie, 217, 9-11. 
Scientific Research Publishing (SCIRP) is one of the largest Open Access journal publishers. It is currently publishing more than 200 open access, online, peer-reviewed journals covering a wide range of academic disciplines. SCIRP serves the worldwide academic communities and contributes to the progress and application of science with its publication.

Other selected journals from SCIRP are listed as below. Submit your manuscript to us via either submit@scirp.org or Online Submission Portal.
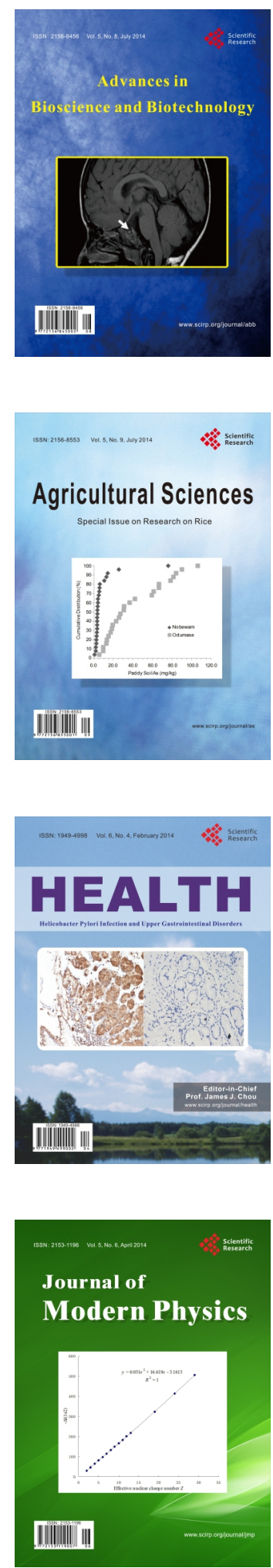
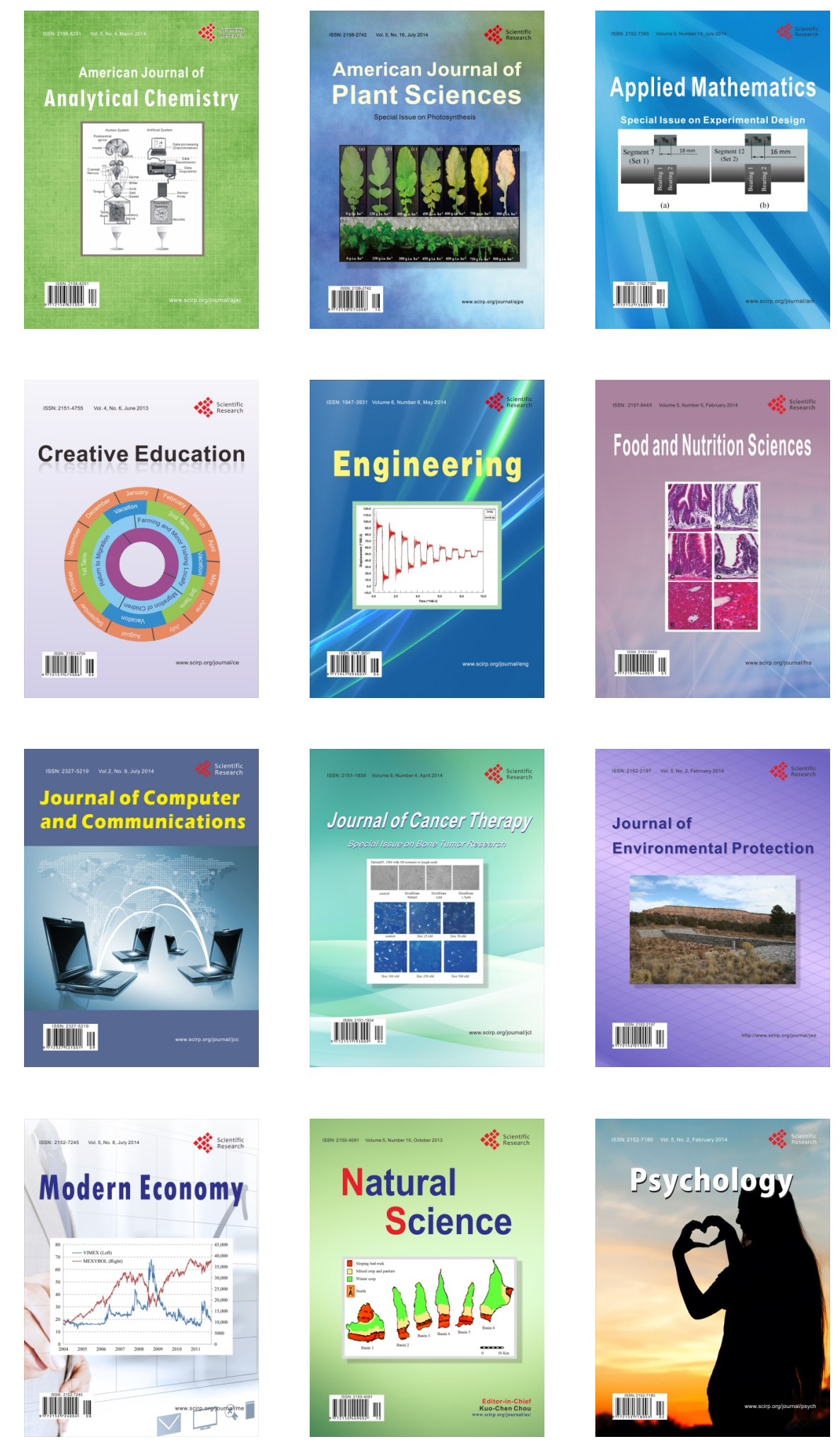